\title{
DOCUMENTS
}

\section{A FRAGMENT OF AN UNKNOWN LATIN VERSION OF THE APOSTOLIC CONSTITUTIONS.}

\author{
(BOOK VIII 4I-END : LAGARDE 274, 26-281. 9.)
}

From a MS in the Chapter Library of Verona ,

LI foll. I $39 b-146 a$

fol. 1396

Feçerit uel inuitus; ac propitius et placidus factus connumeret in ora piorum securitatem po titis, in sinus abrab(am et is)aac 20 et iacob, cum omn $\langle i b u\rangle$ s qui a sae culo placuerunt $\langle e t e\rangle$ gerunt uolumtatem ipsius, $\langle u\rangle$ bi effugit dolor ac meror $\langle e t$ ge $\rangle$ mitus. sur gamus. sese et inuicem sese.per 25 petuo dō per uerbum quod in ini tio
274. 26

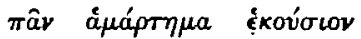

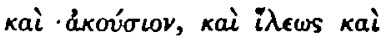

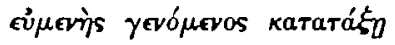

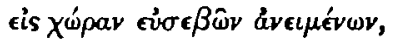

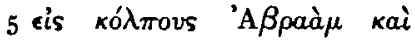

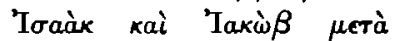

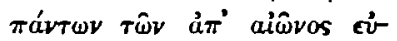

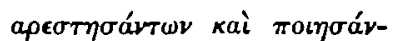

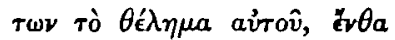

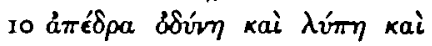

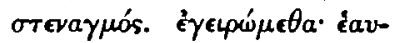

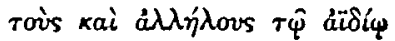

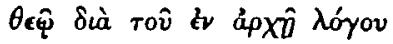

I8. ora appears to be the reading of the MS, or perhaps we should write oras. 18. I9. securitatem potitis; as though the translator was rendering karardip Tois dretelvous.

4. duetufunv y, cf. p. 494 1. I 5 .

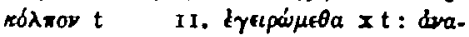
orántes $\mathbf{y}$ 
fol. I $40 a$

conmendemus. et episcopus dicat: qui natura inmortalis ac sine ter mine, a quo omne inmortale ad que mortalem factum est, qui ra 5 tionabile animal hominem $\mathrm{mu}^{-}$ di ciuem mortalem ex confecti onem promisisti, qui enoc et he liam mortis experimentum noconcessisti accipere, DS ABRAHA $^{-}$ IO ET DS̄ ISAAC ET DS SACOB, NON UT MOR TUORUM SED UT UTUENTTUM $D \bar{s} ~ e s$, quod omnium animae aput te ui uunt et IUSTORUM SPिS IN MANU tua sunt quOs NON TANGANT UER

I5 BERA, OMNES ENIm SC̄ITIFICATI SUB TUIS MANIBUS sunt; ipse et nunc inspice in seruum tuum illum quem elegisti et suscepisti in ali si quid inuitus uel uoluntate pec so cauit, et ad angelos placidos ad siste ipsi, ac dignum ipsum facito sinu patriar (charu)m et propheta rum et apostolorum et omniuqui a saeculo tzbi placuerunt, ubi 25 non est meror adque dolor et ge mitus

9. Matt, xxii 32, Luc. $x \times 37,3^{8} \quad 13$. Sap. iii I I5. Deut. xxxiii 3

6. A line has fallen out by homocoleleuton: supply -one fecisti et resurtecti10. non : quorum appears to have fallen out before non. 15. scitificati : perhaps from an exemplar sc̄tificati. 18. Again a line of the exemplar omitted : supply -am sortem et condona illi. 20. ad $I^{\circ}$ : should be omitted: possibly cancelled in the MS. 2I. dignum... facito: the translator read karafíarov for karárałor.
275. 2

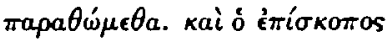

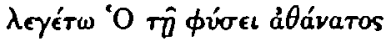

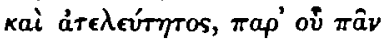

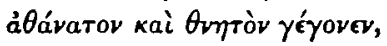

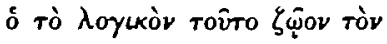

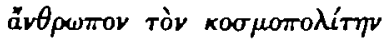

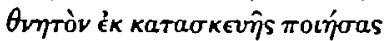

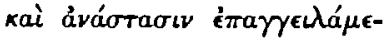
vos, ó Tòv 'Evì̀ кai тòv

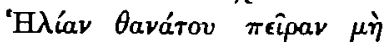

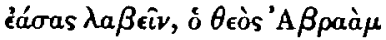

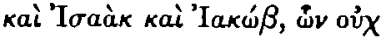

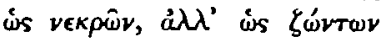

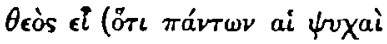

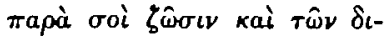

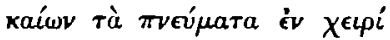

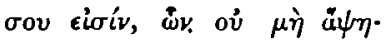

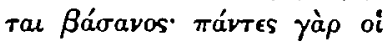

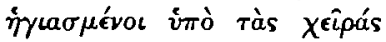

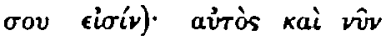

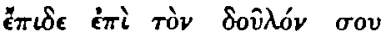

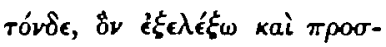

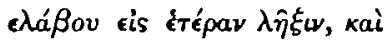

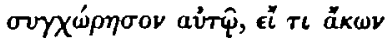

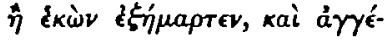

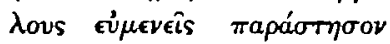

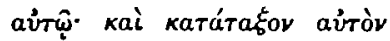

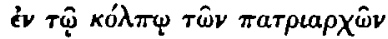

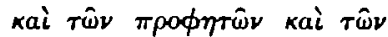

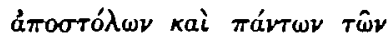

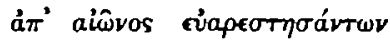

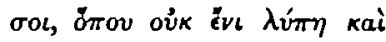

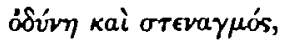

5. Toûto: om. y t 7. Ey кara-

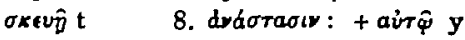

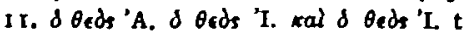

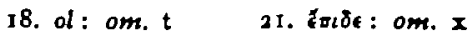
24. tr. entìr thácar $t$ 31. tr. oou eirapeorjodrtor.t 32. nal: om. $t$ 


\section{fol. $140 b$}

sed locus piorum securus el terra rectorum tibi dedicata et illoruqui in ipsa uident gloriam di et sps, per quem tibi gloria honor et ueneratio gratiarum actio et adoratio in $\mathrm{s} \bar{p} \mathrm{u}$ ad saecula amen.

et diaconus dicat: inclinate et benedicimini. et episcopus gra 1o tias agat pro ipsis dicens talia : SER ua $d \tilde{n}$ e POPULUM TUUM AC $b$ ENe DICITO HEREDITATEM TUAMI QUAM adquisisti sc̄o SANGUINE $x \bar{p} i t h i$ HT PASCE IPSOS sub tuam dexteraac tege $i$ psos sub tuas PINNas et da ipsis CERTAMEN conplere bo Num el curSUAI PERFICERE et fideconseruare inmutabiliter sine culpatione SINE INCUSATIONE so per dñm nostrum ihm $x p m$ dilec tum filium tuum, per quem tibi gloria honor ac (uen)eratio in sc̄o spu ad saecula amen. qaes facere connenit

10, 14. Ps. xxvii (xxviii) 9 I1. Act. $1 \times 28$ 15. Ps. xc (xci) 4 , lx (lxi) 516 . 2 Tim.iv 7 I 8 . I Thess. v 23, I Cor. i 8

3. dl et sp̄s: I suspect that the true reading was dI et $x \overline{p i}$. 6 . I have assumed that the Latin version (and the Greek from which it was translated) gave 'in the Spirit', not 'and to the Spirit', compare 1. 22. 9. benedicimini : perhaps the traces which seemed to indicate omnis were really the final letters of (benedic)imini, since omnes is absent from the Greek. 10. serua : the indications appear to point to serua rather than to salua. I1, 12. The text ought obviously to run bene/dicito, but the traces appeared to point to gente-. 13. I should prefer emisti pretioso, as nearer the Greek : but sco seems clear.
275. 18

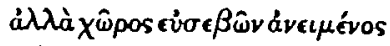

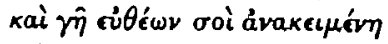

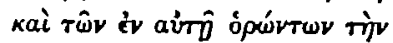

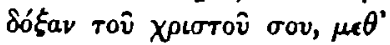

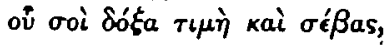

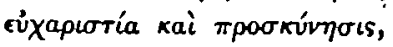

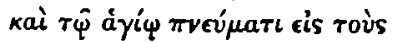
aî̄vas. $\dot{\alpha} \mu \eta^{\prime} \nu$.

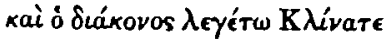

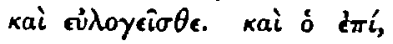

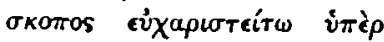

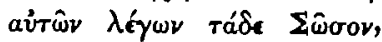

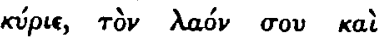

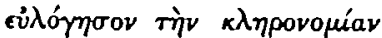

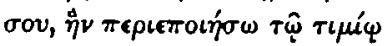

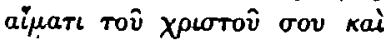

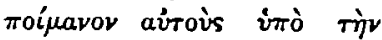

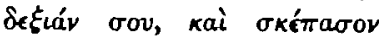

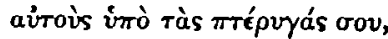

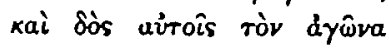

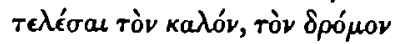

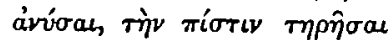

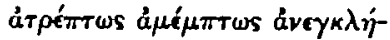

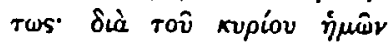

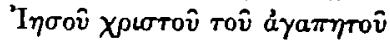

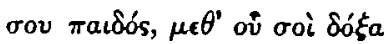

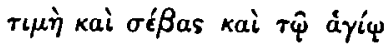

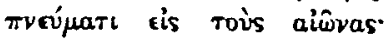
$\dot{a} \mu \dot{\eta} \nu$.

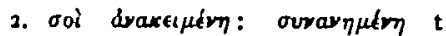
4. $\mu \in \theta^{\prime}$ oi I y: oi oi t 6. кai: om. $t$

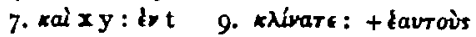
y 12. rabe: rouble $t$ I6. $\mathrm{cal}: \mathrm{om}$.

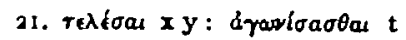

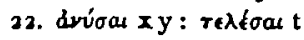


fol. I 4 I $a$

facere autem tertia eorum qui dor mierunt in psalmis et oratione proptet eum qui post tres dies sur rexit, el nona in admonitione

5 uizenfium et eorum qui dormie runt, et tricensima secundum ue terem disciplinam moysepopulus annua pro memoria illius, ac detur.

so ex ipsius fortunis pauperibus $i^{-}$ admonitionem istius. sed haec de piis loquimur, de impiis enim si ta quae mundi sunt pauperibus nihil prode esse

15 cui enim supersti $t i$ inimicum, certum quod et illi si $m i$ grauerit, NoN enim kST iniquitas aput eum. IUSTUS enim dins et iusti TIAS CURauit

20 in memorias autem eorum uocati cum disciplina aepulamini ac di ti more ut qui possitis exorare pro illis qui migrauerunt. presbyteri enim ac diacones $x \bar{p} i$ constituti 25 sobrii esse debetis semper et in se

se

$$
\begin{aligned}
& \text { 7. Deut. xxxiv } 8 \\
& 16 \text { 17. Ps. } x(x i) 7
\end{aligned} \text { 17. Ps. xci(xcii) }
$$

6. I had ventured, following some slight indications of the MS, to restore tricesima to the tert in accordance with the reference to Deuteronomy, against

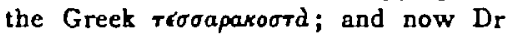
Spagnolo confirms my conjecture from a second inspection of the MS. 7. I am not satisfied with disciplinam for rúzov: perhaps imaginem.

$$
276.3
$$

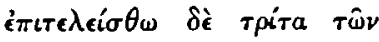

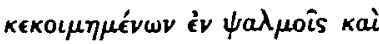
$\pi \rho 0 \sigma \epsilon X^{a i ́ s ~ \delta i a ̀ ~ \tau o ̀ v ~ \delta i ̀ ̀ ~ \tau \rho i \omega ̂ v ~}$

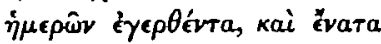

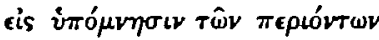

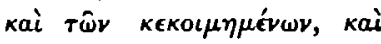

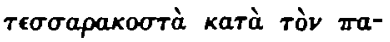

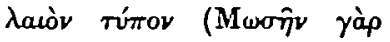

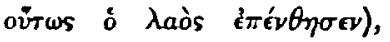

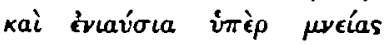
aủrov̂ кai $\delta \delta \delta ́ \sigma \theta \omega$ í

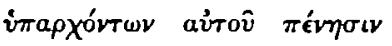

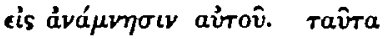

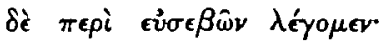

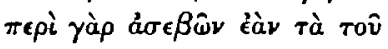

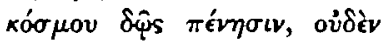

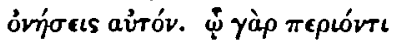

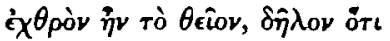

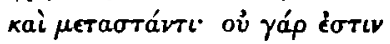

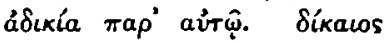

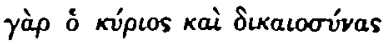

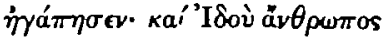

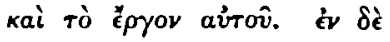

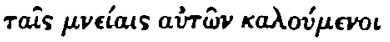

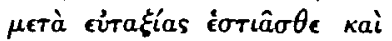

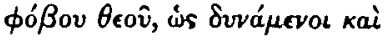

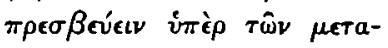

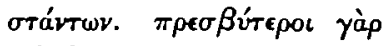

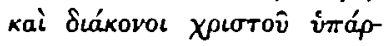

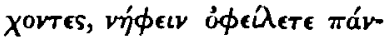

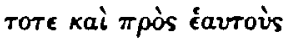

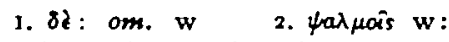

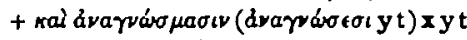
Lagarde 3. Tdu did: om. perhomoro-

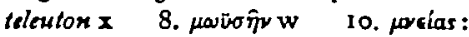

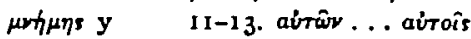
... aurầ w . II.

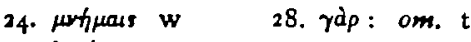
3о. oqfítitтu $x$ 


\section{fol. 14 I $b$}

et in alios ut possitis inmoderatos docere. dicit autem scribtura po te TENS FERUORE CONCITANTUR : UINUM NE POTENT, UT NE POST QUAMI UIBERINT

5 OBLIUISCANTUR SAPIENTIAM ET ReC TE IUDICARE NON POSSINT. igitur et presbyteri ac diaconi post deum omnipotentem ac dilectum filiuipsius potentes sunt aeclesiae. ro hoc enim dicimus non ut minime uibant (aliter enim iniuriam est facere di facturae quae in laetitia est) sed ne uino uerint. non. enim scribtura dixit minime po

I 5 tare uinum sed quid ait? UINUs NON UIBERE IN EBRIAETATEM, $\mathrm{e}(\boldsymbol{t}$ ite TUM SENTES PULLULANT IN MTA ( $m$ i EBRI. hoc autem non de cleric $\langle$ is $\rangle \mathrm{di}$ cimus tantum sed de omni etiam

20 plebeio xpiano super quo inuoca. tum est nomen dñi nostri ihū $x \bar{p} i$; et ipsis enim dictum est cUI UE? cUI TU MULTUS? CUI TEDIUM SOLLICITUDO? CUIUS LIUIDI OCULI? CUI CONTRITIONES 25 SUPERUACUE? NONNE EORUM QUT

2. Prov. $x \times x i 4,5(x \times \vee 72,73)$ I2. Ecclus. Ixxiv 27 (xxxi 35) I5. Agg. i 6 17. Prov. xxvi 9 22. Prov. xxiii 29, 30

4, I 1, 16. uibere for bibere. 7. deum : it is most unusual, almost unique, to find this word written in full in a MS, however early, though in inscriptions it is much less uncommon.

I 2. factura cad.
276. 18

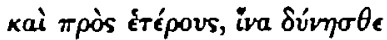

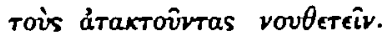

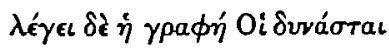
$\theta u \mu \omega ́ \delta$ eıs cioiv. olvov $\mu \grave{\eta}$

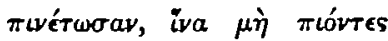

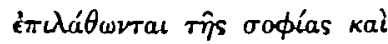

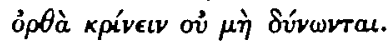

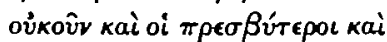

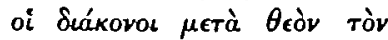

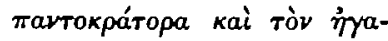

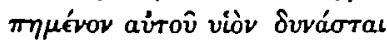

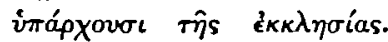

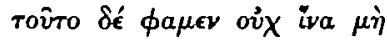

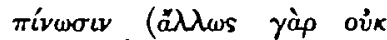

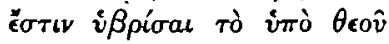

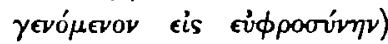

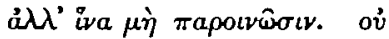

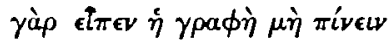

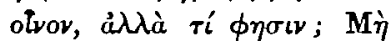

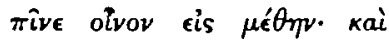

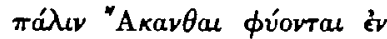

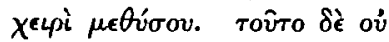

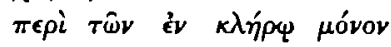

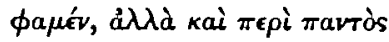

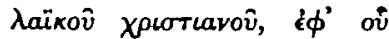

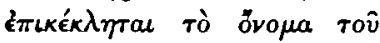

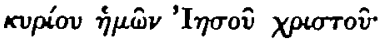

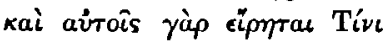

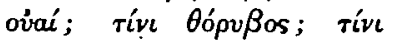

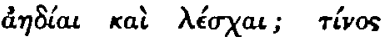
$\pi \epsilon \lambda \omega i$ oi $\delta \phi \theta a \lambda \mu o i ́$; tívi

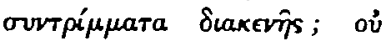

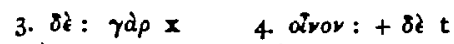

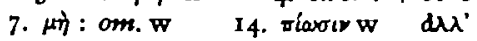
is $\mathrm{y}$ oik w y : cal $\mathrm{t}$ : om. $\mathrm{x} 20$. riveir wo 27. aupiou: + tôे $\theta \epsilon o \hat{~} w$

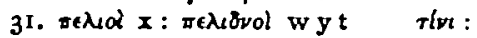
rívos $w$ 
fol. $142 a$

TARDANT IN UINO ET EXPLORANTTUM

UBI CONUTUA FTUNT

de illis qui persecuntur et fugiunt propter

fidem quod anxilinm adipiscentur

5 Persecutos propter fidem et urbeex urbem fugientes quad memo ria ha)bent dñi uerba suscipi ; scien

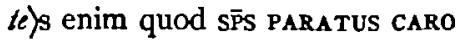
autc) M DEBILITATE LABORAT, fuginnt 10 ac rapinam substantiae propo nun)t ut innegatum in sese nome ${ }^{-}$ $x \bar{p} i$ conseruent. ministrate igitur ipsis quae ad usum, mandatum do minicum implentes. quod unusquis ${ }_{15}$ que in qua destinatus est sorte de

bet contemptas ordinem oustodi re sed minime sepere sibi quae non sunt conmissa. hoc autem ones conmuniter edicimus unum

20 quemque contemptum esse or dine sibi dato et non transire li mites, non enim sunt nostri sed di sunt. QUI AUDIT ENMI UOS d̄̄s ait ME AUDIT ET QUI ME AUDIT AUDIT 25 BUMI QUI ME MISIT ET QUI UOS CON

TEMINIT

5. Cf. Matt. $\times 23 \quad$ 8. Matt. xxvi 41 - Marc. xiv $3^{8}$ 33. Luc. $x$ 16, Matt. $\times 40$

2. conuiua $\operatorname{cod}^{*}$, conuiuia $\operatorname{cod}^{2}$. 7-9, I I. Some letters are lost at the beginning of each of these lines; cf. fol. $b$. 7. sus-

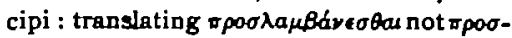

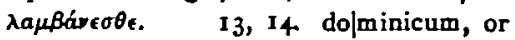
in 1.14 dñicum. 16, 20. Read of course contentus, contentum.
$277 \cdot 7$

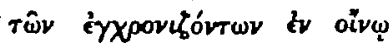

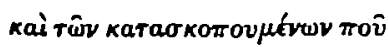
tótol Yívovtal;

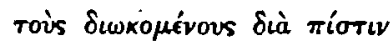

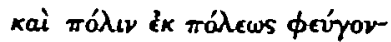

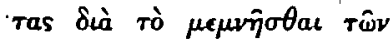

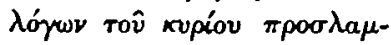

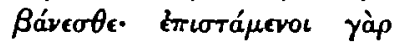

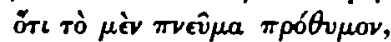

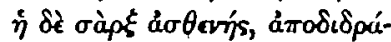

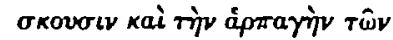

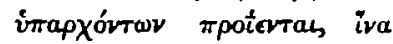

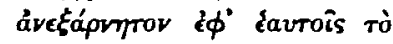

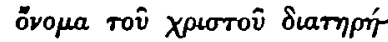

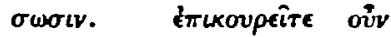

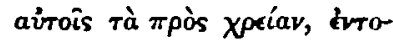

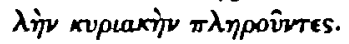

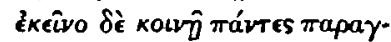

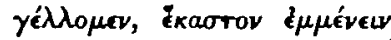

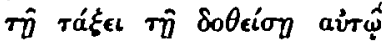

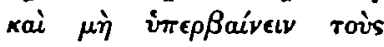

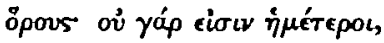

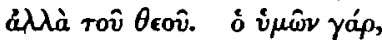

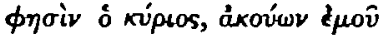

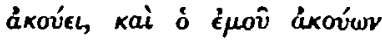

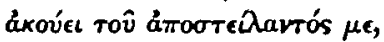

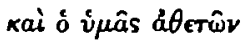

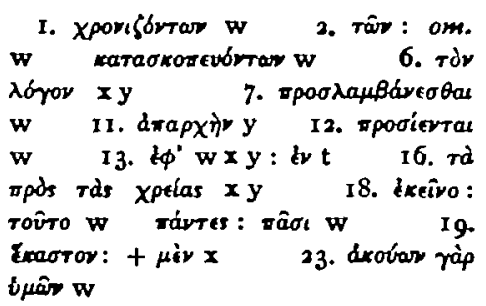




$$
\text { fol. } 142 b
$$

BIE CONTEMNIT QUI AUTEM ME $\mathrm{CO}^{-}$ TEMNTT CONTEMNTT EUH QUI HR MI sIT. si enim inanimata facta or dinem reddit, qualis est nox dies 5 sol luna sidera aelementa ui ces in uices menses septima nae dies orae, ac seruiu(nt usui qui inpositus est secundum quod dictum est DEFINITION<em 10 posuisti QUaH NON TRANSEANT et iterum POSUI AUTEM IPSI LIMITES ET CIRCUMDRDI CLAUSTRA ET POR TAMI, DIXI AUTEM IPSI USQUE HUC UENIES AC NON TRANSIES ; quanto 15 magis uos debetis nihil audere transmouere de illis quae uo bis di uoluntate a nobis sunt constituta? sed quoniam con temptibile multi et hoc duxerunt so esse, confundere uero ordines et ordinationem quae est in uno quoque eorum transmouere audent, adripientes sibi digni tates non datas et permitten 25 tes sibi tyrannico more quora

$$
\begin{aligned}
& \text { 9. Ps. ciii (civ) } 9 \text { I I. Job xxrvii } \\
& \text { I0, I I }
\end{aligned}
$$

3. inanimata : perhaps inanimalia cod. 4. reddit : singular, because of the Greek verb drooq'sat, after the neuter plural.
277.20

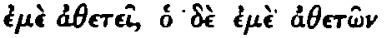

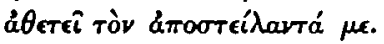

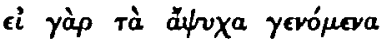

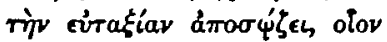

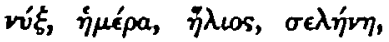

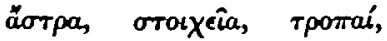

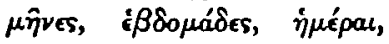

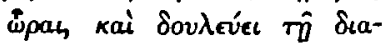

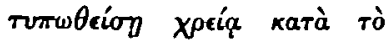
cipquévov "OpLv है $\theta$ ov, 8 ovं

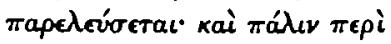

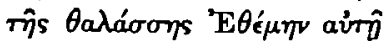

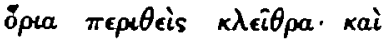

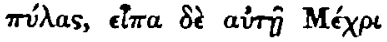

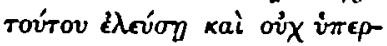

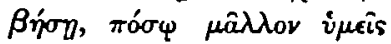

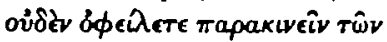

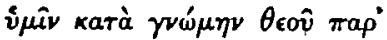

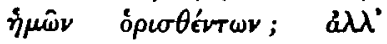

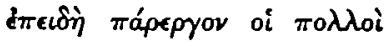

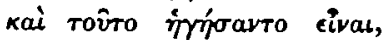

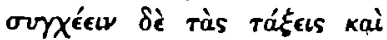

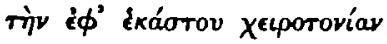

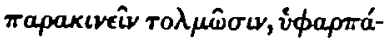

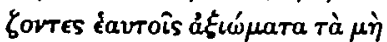

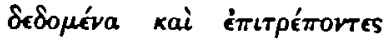
Éavtoís Tupavvikîs

I. $1 \mu \lambda \mathrm{I}^{\circ} \ldots \mathrm{d} \theta \mathrm{eTây}$ : om. perhomono-

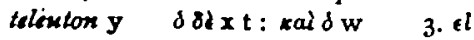
rip: deficit abhine w ràp $\mathrm{t} t$ : tolvury

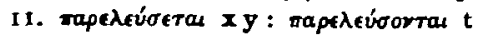

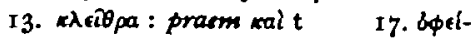

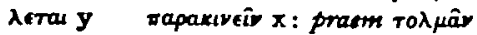
yt 26. 8.66utsat 


\section{fol. I $43 a$}

non habent potestatem dare, et proplerea $d \overline{\mathrm{m}}$ in iram conmouent, quemadmodum coreitae $a c$ rex ogias dignitatem inuase

5 runt pontificatum preter dì uo luntatem, ac facti sunt illi qui dem

facie leprosus el stimulant quideeum qui disposuit xpm et con ro tristant et spm scm infirman tes ipsius testimonium repente inminens periculum talia facientibus prouidenies et illam quae in sacrificia et grati

I5 arum actiones desidiam ex eo quod ab illis qui minime deueant offerantur impie, lusum existi mantes pontificalem dignilatequae imitationem continet mag so ni pontificis $i h \bar{u} \times \bar{p} \mathrm{i}$ regis nostri,

- necessitatem habuimus docere. iam enim QUIDAII DE RECTO ITINE RE DISCESSERUNT SECUTI pIOpria ${ }^{-}$ uanitatem. dicimus autem quod 25 moyses di cultor cui ds̃ CORAM

7, 9. Num. xvi 35,30 xxvi I 9 xxxiii II

4. oxias dignitatem : the word praeter or contra appears to have fallen out here, corresponding with the Greek rap' oflay. 5. preter dI uoluntatem: Greek arcu

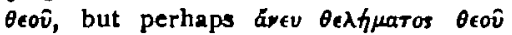
stood in the translator's text.
278.8

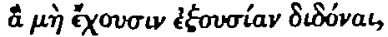

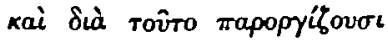

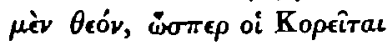

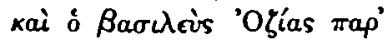

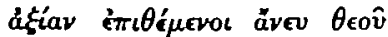

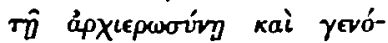

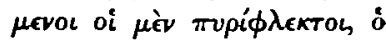

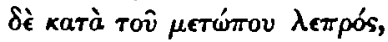

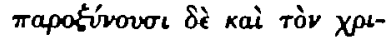

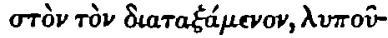

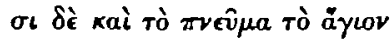

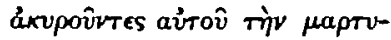

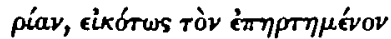

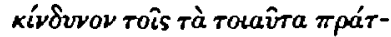

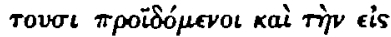
ràs Ourías kaì cúxaplotías

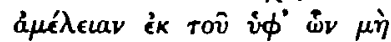

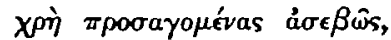

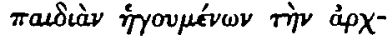

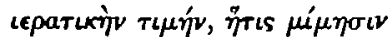

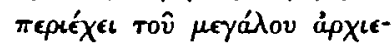

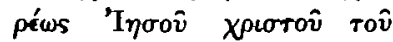

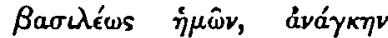
"̈

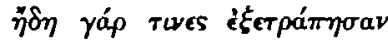

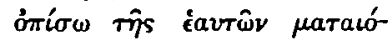

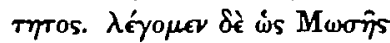

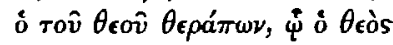
ह่vér

I. Ixavor t 9. $\mathrm{xal}+\partial y \ldots \lambda v-$

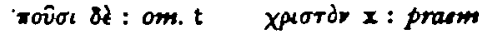

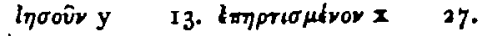
it: om.t 


\section{fol. 1436}

cum PRAESENTI loquebatur SI QUIS AD SUUN LOQUATUR AMITUM CUI di cit SCIO TE SUPRA OMNES CUI IN ORE loquebatur ET NoN PER incerta 5 uel per somnia uel per angelos uel per pau as, iste quando in ebat diuinam sanctionem diuisit quae debeant quidem a potifcibus celebrari quae uero 10 a sacerdotibus quae autem a di aconibus, et unicuique propriaet conuenientem ministerio tribuit religionem. et quae pon tifices quidem praeceptum est I5 celebrare, haec sacerdotes infadum erat haec diaconi non adi bant, sed unusquisque quae acce pit ministeria circumscripta ser bat: si quis ultra quam traditum so est adire uolebat, mors erat poe

na. hoc autem maxime demons trabit quae fuit in saul experien tia; qui sacrificasse sine prophe ta ac pontifice samuel existima 25 tus intulit sibi peccatum execra tionem

3. Exod, xxxiii i 7 4-6. Num, xii 6, 8 22. I Reg. xiii 8 sqq.

I. loquebatur : ut has apparently dropped out after -ur. 6. pauras was Dr Spagnolo's first transcription, and he still on second reading adheres to five letters of the six. I cannot tell

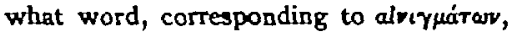
this can stand for.

7. sanctionem ; or perhaps the MS has pactionem 'covenant'. 18, 19. ser|bat : should of course be seruabat. 22. demonstrabit : for demonstrauit. 25 . execrationem : et has apparently dropped out before ex.
278,2 I

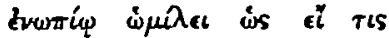

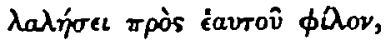

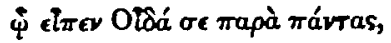

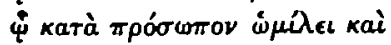

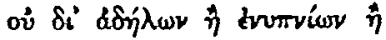

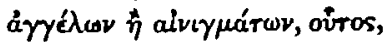

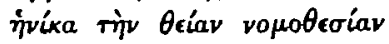

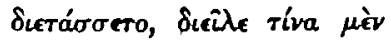

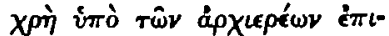

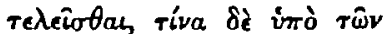

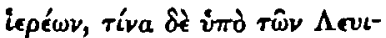

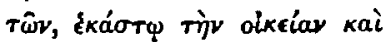

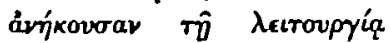

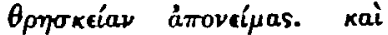

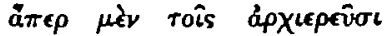

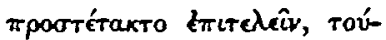
rols roìs iepéas ov $\theta$ ecutòv

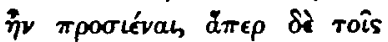

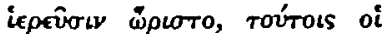

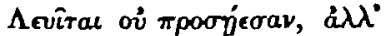

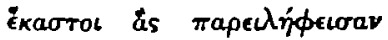

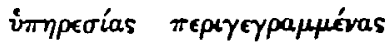

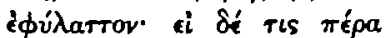

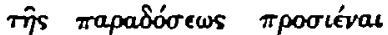

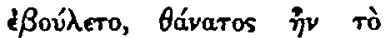

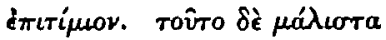

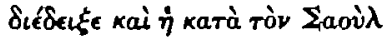

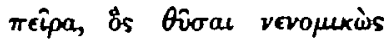

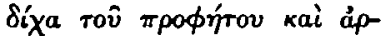

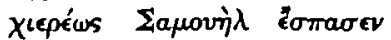
cis Éavtòy àuaptíav кai katápav

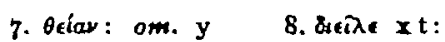

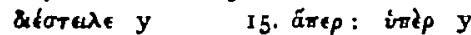

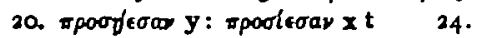

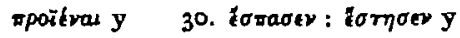


fol. $144 a$

nullo modo abiciendam ac neq' ob id quod ab ipso erat litus exora tus est profeta. euidentissimo au tem actu demonstrauit ds secun 5 dum oziam quod non in latione super iniquitate poenas adiecit, et qui pontificatum furiose calca uit et regno pribatus est. quae ue ro de nostris temporibus forte noro ignoratis, scitis enim forte a no bis episcopos nominatos ac pres byteros ac diaconos ora $\langle a\rangle$ ne ac manibus inpositi(s) nominum diuersitatem sign(if)icantes. non

$t_{5}$ enim ILLE QUI UOLHBAT aput nos Ma NUY IMPLEBAT, quemadmodum in notho uitularum sub hieroboacircumcisi sacerdotii, sed Uoca TUS A Dō. si enim non erat quidem so lex quidem et hordinzm diuersi tas, sufficiebat per unum nomeomnia fieri; sed a xp̄o edocti seque ${ }^{-}$ tiam rerum, episcopis quidem ea quae sunt pontificatus tribuimus 25 presbyteris autem ea quae sunt sacerdotii

5. 2 Paralip. xxvi 16 sqq. $15 \cdot 3$ Reg. xiii 33, xii 28 sqq. I8. Heb. $v_{4}$

2. litus : from linere 'to smenr'. 5. in latione $m$. I, in dilatione $m$. 2 . 10 . forte : read perhaps certe. 13. nominum : after this word the words diuersitate [et] rerum have apparently dropped out by homocourcton, so that the phrase would run 'nominum diuersitate [et] rerum diuersitatem'. I 7, I8. The transletor has quite misunderstood the meaning of the Greek 'in the case of the spurious counterfeit priesthood of the calves under Jerobonm '. 19, 20. quidem ... quidem cod: in the second of the two places we must read quaedam.
279. 7

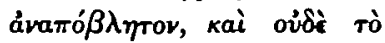

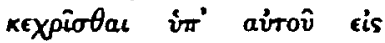

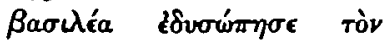

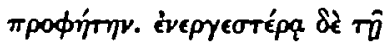

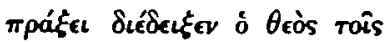

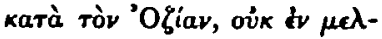

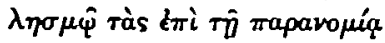
ciompakáperos díkas kai ò

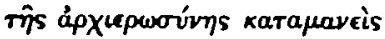

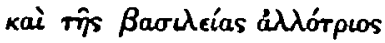

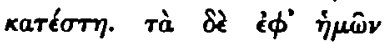

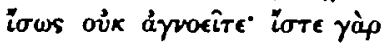

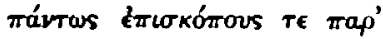

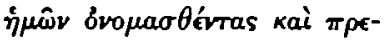

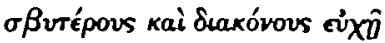

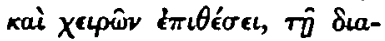

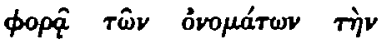

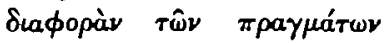

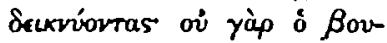

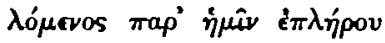

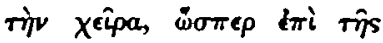

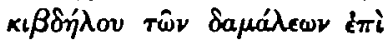

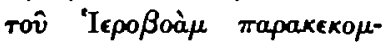

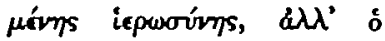

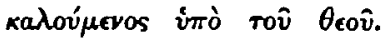

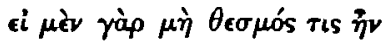

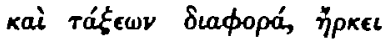

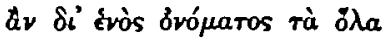

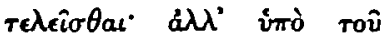

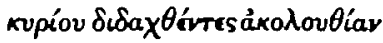

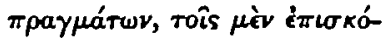

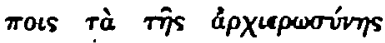

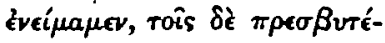

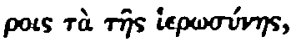

5. Reffer $y$ rois $x:$ ty tais $y t$ 8. wail \& $x t:$ o ois $y$ I\} TEI: om. yt I7. Tiv $x$ : pram wail yt 20.

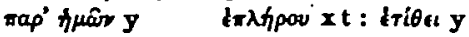

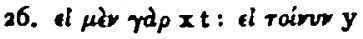


fol. $144 b$

diaconis uero quae ad utrosque ministeria, ut pure peragantur

ea que ad religionem pertinent. neque enim diacono offerre fas

5 sacrificium aut baptidiare aut benedictionem parwam uel magnam facere, neque presby terum ordinationes facere. non enim sc̄m est ordinem euerti: NoN EST ENIM DS INCONSTANTIAE Ut in feriores quae sunt superiorum tyrannico more proprient nouasanctionem fingentes in malo suo nescientes quod durum ipsis

15 ADURRSUS STTHULOS CaLCARE. non enim nos aut episcopos oppugnant huiusmodi homines sed omni um episcopum ac patris pontifi cem $x \bar{p} m$ ihm $d \bar{m}$. a moyse quide ${ }^{-}$ so dō amicissimo ac pontifice cons tituti sunt pontifices et sacerdo tes et diaconi, a saluatore nostro tredecim nos apostoli, ab aposto ${ }^{*}$ lis autem ego clemens ego iacob ${ }_{25}$ et nobiscum alii ut ne iterum om nes

9. I Cor. riv 33 I5. Act. xxrvi 14

20. ac pontifice: Moses is not called ' high priest' in our present Greek text, but cf. $145 b$ l. 24, which seems to shew that the Latin is right here. 22. Note the tranglation of Arveitar, even where Old Testament Levites are meant, by diaconi: cf. p. 500 l. 16.24 Note the inversion of order, by which Clement precedes James in the Latin text.
279. 23

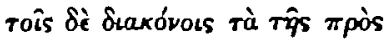

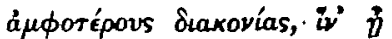

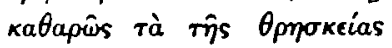

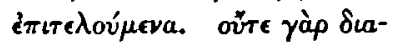

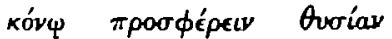

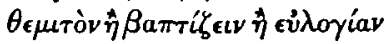

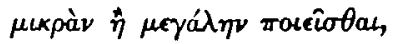

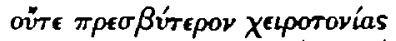
ė $\pi \iota \tau \epsilon \lambda \epsilon \hat{\omega}$. ở $\gamma \grave{\alpha} \rho$ ó $\sigma \omega \nu$

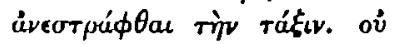

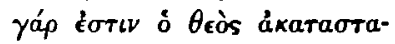

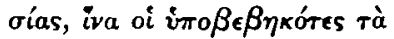

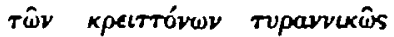

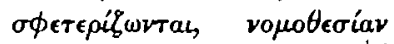

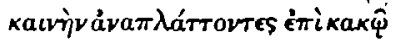

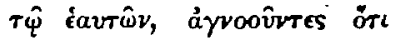

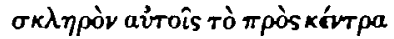

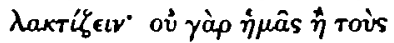

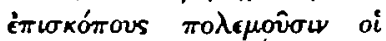

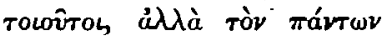

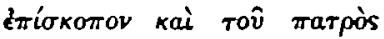

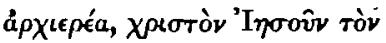

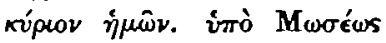

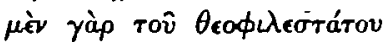

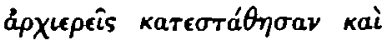

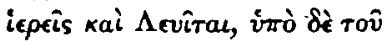

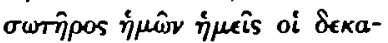

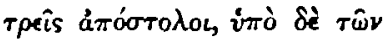

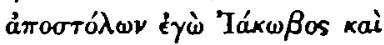

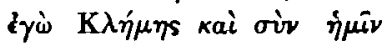

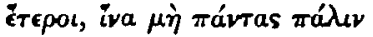

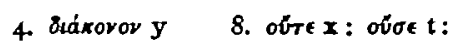

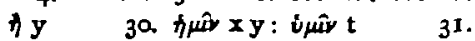
xúvtax tr. vúlesy rámas y 
fol. $145^{a}$

connumeremus, conmuniter au

tem a nobis omnibus presbyteri ac diaconi et lectores. primus igi tur natura pontifex unigenitus

$5 \times \bar{p} s$, NON SIBI HONOREM adripiens SED A patre constitutus; qui ho $\mathrm{m}\langle o$ f actus propter nos ac spirita le (sacr)ificium offerens dō suo ac $\mathrm{pa}\langle$ tri a)nte passionem, nobis cons Io titu(it so)/IS HOC FACERE, aliis quidenob iscum) constitutis qui in ipsuc(rediderunt, s)ed tamen non omnis $q\langle u$ icredidit $i$ )am et sacerdos cons titutus est $e t$ pontificalem honorem ${ }_{5}$ inpetrabit. post autem elebati onem ipsius nos optulimus secun dum constitutionem ipsius SACRI FICIUM PURUH adque insanguinenet episcopos ordinauimus ac praes 20 byteros et diacones numero sep tem. ex quibus unus erat stefanus beatus martyr non deficiens a no bis eum qui in $d \bar{m}$ est bonus sensus qui tantum di culturam fide oste ${ }^{-}$ 25 derat et ea quae dñm est nostro

5. Heb.v4,5 6. Symbolum Io. I Cor. xi 24, \&ce. 17. Mal. i i

3. Subdeacons are omitted in the Latin text. 5. adripiens : I supply the word by comparison with 14561 . 21 . 14. The line is too long as it stands; but I do not see how to shorten it. 15 . inpetrabit for inpetrauit. Note the term 'elevatio' for the Ascension. 23. eum : the word secundum must have dropped out before eum. 25. ea : should be eam. quae: ad must have dropped out before dominum.
280.9

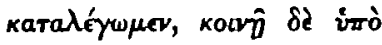

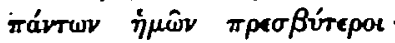

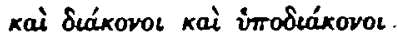

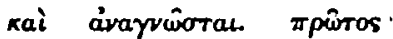

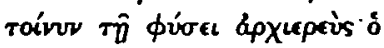

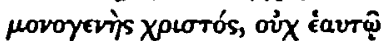

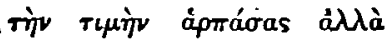

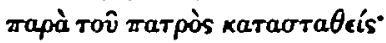

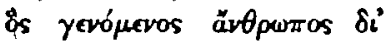

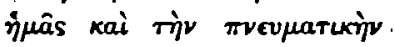

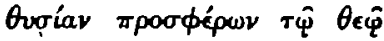

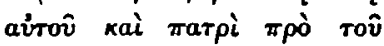

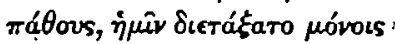

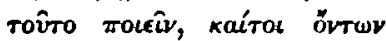

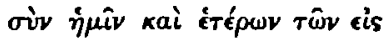

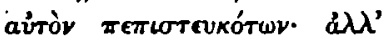

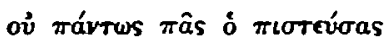

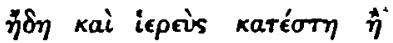

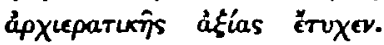

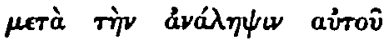

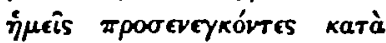

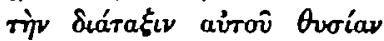

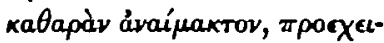

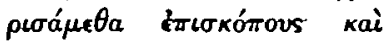

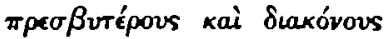

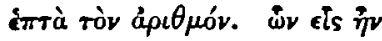

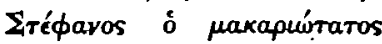

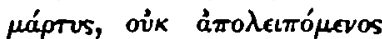

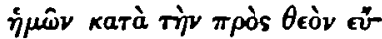
volay. के roбôेtov Tiेv $\theta$ co

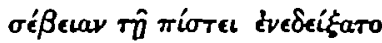
кai Tìn cis tòv кúptov ì $\mu \hat{\omega} v$

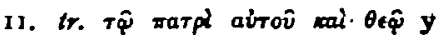

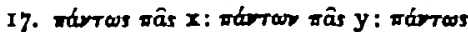

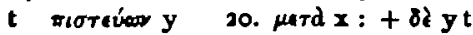

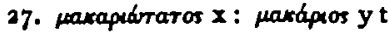




\section{THE JOURNAL OF THEOLOGICAL STUDIES}

\section{fol. $145 b$}

ih $\bar{m} x \bar{p} m$ dilectionem ut pro ipso daret etiam animam ab interfectorib' dni lapidibus perculsus iudeis : sed tamen talis ac tantus úir qui $5 \mathrm{~S} \bar{P} U$ FER URNS $\mathrm{x} \overline{\mathrm{p}} \mathrm{m}$ uidens $\mathrm{n}$. DEXTRIS Dī et caelestes portas APRRTAS nusquam inuenitur illis (quac) noad diaconii ministeriz( $m$ pertine) bant usus aut quod sact(ifico)um 10 retculit aut quod man(us a)licui inposuit sed dia(conii ordi) ne obseruarit usqu(e in finem sic) enim decebat $x \bar{p}(i$ martyr $)$.mo derationem reseruare. si uero

I5 filippum diaconum et annaniafidelem fratrem incusant aliq̧i quod unus quidem eunuchum baptizauit alter uero me paulum, ignorant ipsi quod dicimus. nos 20 diximus eis quod NON SIBI QUTS arripit sacerdotii dignitatem sed uel a dno accipit, quemadmo dum melchisedech et iacob, aut a pontifice, ut aaron a moysen : 25 igitur et philippus et annanias no-

se se

5. Act rviii 25 (cf. Rom. Iii II) 5,6. Act. vii $55,5^{6} \quad$ 17, 18. Act. viii. $3^{8}$, ix 18 20. Heb. $v_{4}$

I. ispo for ipso cad. 20. eis : read enim. 23. Note Iacob in the Latin, where the Greek (rightly, I suppose) hes Iob.
280. 24

'I

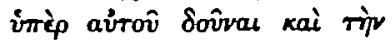

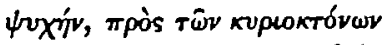
'Iovfaíwr $\lambda i \theta 0$ s $\beta \lambda \eta \theta$ eís.

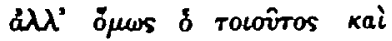

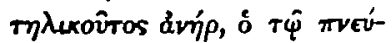

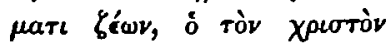

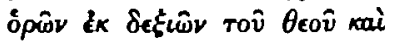

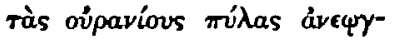

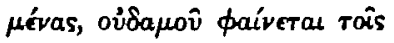

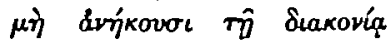

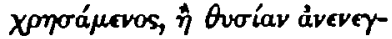

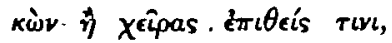

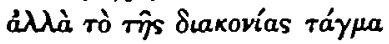

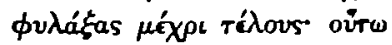

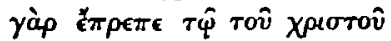

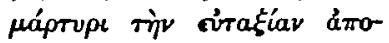

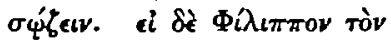

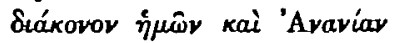

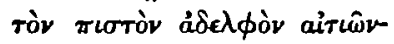

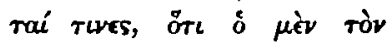

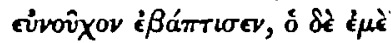

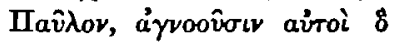

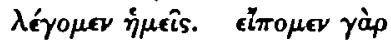

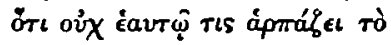

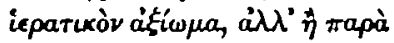
Tov̂ $\theta \epsilon o v ̂$

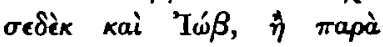
apxiepéws iss 'Aapìv mapà

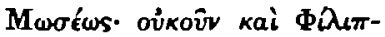
ros kai 'Avavías

29. dpxicptar y 
fol. $146 a$

protulerunt, sed a $x \bar{p}$ o promoti sunt pontifice dì incomparabilis.

\author{
28I. 7 \\ oủx éavtoìs $\pi \rho$ ofillovtó,

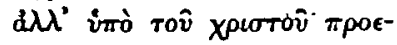

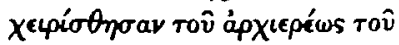

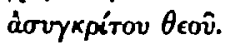

I. Lavrois: praem aúroi $y$
a. Spagnolo.
C. H. Turner.

\section{INTRODUCTION TO THE FRAGMENT PRINTED ABOVE, PP. 492-505.}

Lagarde's text of the Apostolic Constitutions rested on four MSS, w (Petersburg gir. 254 ; A. D. I I I I), x (Vienna hist. gr. 46 ; saec. xiv), y (Vienna hist. gr. 47 ; saec. xvi), and $z$ (Paris gr. 93I ; saec. xvi); but of these $z$ is wholly, and $w$ partially, defective for the part corresponding. to the Verona fragment. Besides his MSS he wisely employed the editio princeps of Fr. Turrianus (Venice, A. D. ${ }^{1} 5^{6} 3$ ) as an additional witness, under the symbol $t$; for Turrianus used three MSS, and these were all of them older at least than $y$ and $z$, since they could already in

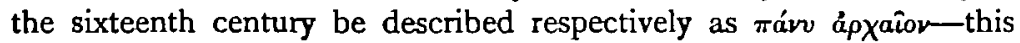
one was from the monastery of Patira at Rossano in Calabria-another кai

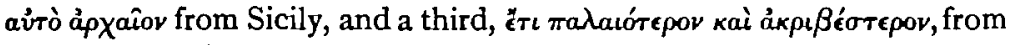
Crete: it was this Cretan MS which, as the oldest and most correctly written, he had (he tells us in his preface) followed almost exclusively. Lagarde does not rate his predecessor's work very highly; and it is true that, for instance, either Turrianus's MSS or Turrianus himself corrected the paraphrase of 2 Tim. iv 7 in the bishop's thanksgiving (p. 494 1l. r6, 77 above) into accord with the New Testament text. But on the other hand, Turrianus agrees with our Latin fragment against Lagarde in readings that are certainly original, especially in the doxologies.

In order to facilitate comparison with the Greek, I have printed Lagarde's text and a selection from his apparatus ${ }^{1}$ parallel with the Latin ; but the reader should note that on one occasion, p. 495 1. 2, the

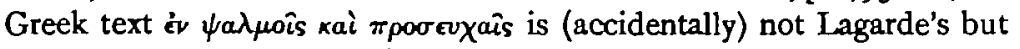
that of the Greek MS which appears to agree with the Latin. The Latin does not side systematically with any one of the Greek authorities; but it does side definitely against the peculiar readings of y, e.g. 492.23

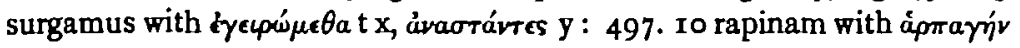

' I have added, however, several more biblical references than are marked in his text. 